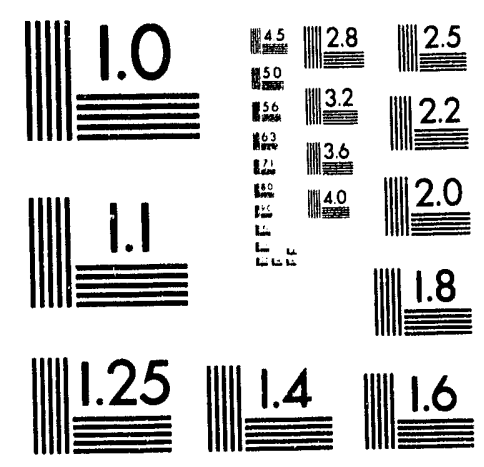



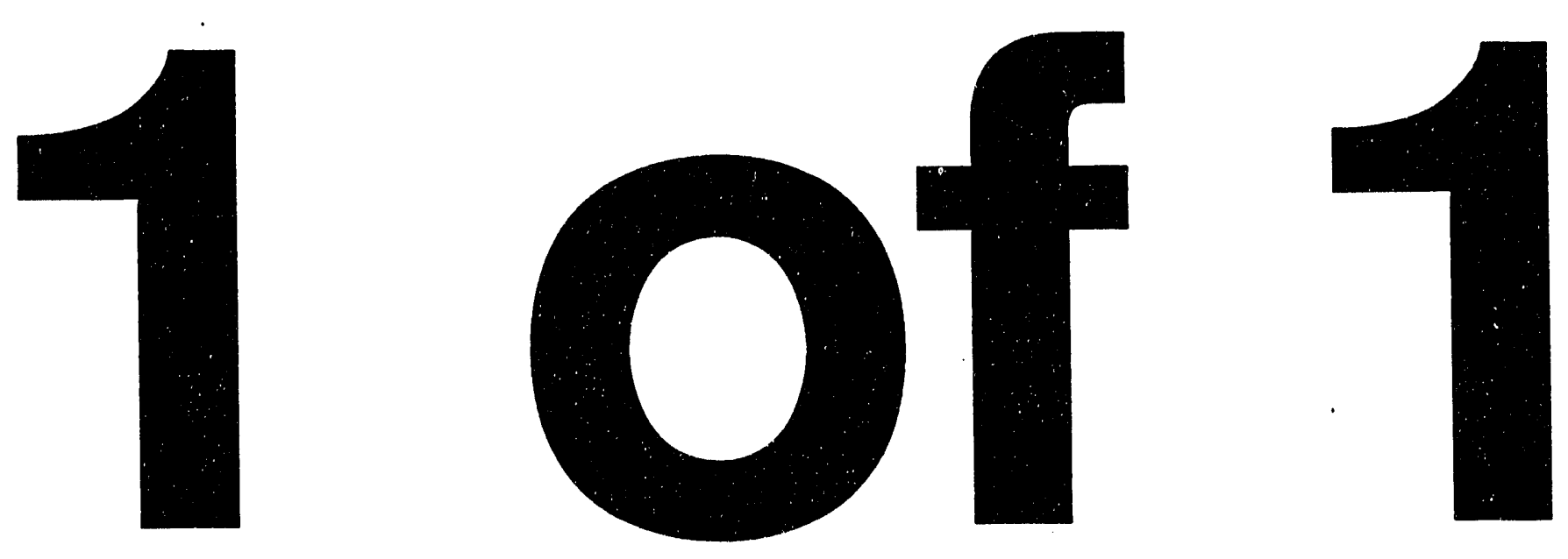


\title{
Design of the ALS Transverse Coupled-Bunch Feedback System
}

\author{
W. Barry, J.M. Byrd, J.N. Corlett, J. Hinkson, J. Johnson, \\ G.R. Lambertson, and J.D. Fox
}

\author{
Accelerator and Fusion Research Division \\ Lawrence Berkeley Laboratory \\ University of California \\ Berkeley, California 94720
}

May 1993

This work was supported by the Director, Office of Energy Research, Office of Basic Energy Sciences, Materials Sciences Division, of the U.S. Department of Energy under Contract No. DE-AC03-76SF00098. 


\title{
Design of the ALS Transverse Coupled-bunch Feedback System*
}

\author{
W. Barry, J. M. Byrd, J. N. Corlett, J. Hinkson, J. Johnson, G. R. Lambertson \\ Lawrence Berkeley Laboratory, Berkeley, CA 94720 USA \\ J. D. Fox \\ Stanford Linear Accelerator Center, Stanford, CA 94309 USA
}

\section{Abstract}

Calculations of transverse coupled bunch growth rates in the Advanced Light Source (ALS), a $1.5 \mathrm{GeV}$ electron storage ring for producing synchrotron radiation[1], indicate the need for damping via a transverse feedback (TFB) system. We present the design of such a system. The maximum bunch frequency is $500 \mathrm{MHz}$, requiring that the FB system have a broadband response of at least $250 \mathrm{MHz}$. We describe, in detail, the choice of broadband components such as kickers, pickups, power amplifiers, and electronics.

\section{INTRODUCTION}

Because of the high beam current and large number of bunches at the ALS, coupled-bunch instabilities are predicted to be one of the limiting factors in achieving the design beam intensity while maintaining good beam quality[2]. The instabilities are driven by the $\mathrm{RF}$ cavity dipole higher order modes (HOMs) and the resistive wall impedance of the vacuum chamber. We have designed a TFB system to damp beam oscillations to an acceptable level. General ALS parameters relevant to the system are listed in Table 1.

\section{FEedback Requirements}

The proposed TFB system is a so-called bunch-by-bunch system because each bunch is acted upon independently. This requires that the system bandwidth be at least one half the bunch frequency to encompass all possible coupled-bunch mode frequencies. This bandwidth requirement poses the most difficult design restrictions on the system.

The TFB system can, in principle, be operated in any one of many frequency bands $(0-250 \mathrm{MHz}, 250-500 \mathrm{MHz}$, etc.). In order to minimize expensive wideband highpower driver amplifier power, we chose to kick in the 0-250 $\mathrm{MHz}$ frequency band, where the impedance of a striplinepair kicker is greatest. In addition, the fastest growing CB modes are driven at low frequency by the resistive wall impedance, where the kicker impedance is maximum. The lowest frequency unstable mode is assumed to be no lower than $150 \mathrm{kHz}$.

The amount of voltage kick/turn and gain required in the system is determined by the driving impedances, total beam current, and transverse oscillation amplitude. In order to damp an oscillation, the TFB system must be capable of providing a transverse voltage kick equal to the

* This work was supported by the Director, Office of Energy Research, Office of Basic Energy Sciences, Materials Sciences Division, of the U.S. Department of Energy under Contract No. DE-AC0376 SF00098.

\begin{tabular}{clc}
\hline \hline Parameter & Description & Value \\
\hline$E_{b}$ & Beam energy & $1.5 \mathrm{GeV}$ \\
$C$ & Circumference & $196.8 \mathrm{~m}$ \\
$f_{r f}$ & RF Freq. & $500 \mathrm{MHz}$ \\
$N$ & Number of bunches & 328 \\
$I_{0}$ & Total DC beam current & $0.4 \mathrm{~A}$ \\
$\sigma_{\ell}$ & RMS bunch length & $3.9 \mathrm{~mm}$ \\
$Q_{x, y}$ & Horizontal betatron tune & $14.28,8.18$ \\
$\beta_{x, y, c a v}$ & $\beta_{x, y}$ at cavity & $11.5 \mathrm{~m}, 5 \mathrm{~m}$ \\
$Z_{\perp, R W}$ & max. RW impedance & $2 \mathrm{M} \Omega / \mathrm{m}$ \\
$Z_{\perp, H O M}$ & max. HOM impedance & $4 \mathrm{M} \Omega / \mathrm{m}$ \\
\hline \hline
\end{tabular}

Table 1: ALS parameters used for calculations.

beam induced voltage. Neglecting radiation damping, this roltage is given by

$$
V_{b}=I_{0} \Delta x Z_{\perp}
$$

where $\Delta x$ is the transverse oscillation amplitude of a coupled-bunch mode.

- Kicker power is provided to deliver a transverse kick of $1.6 \mathrm{kV} /$ turn; this will damp a CB mode oscillation with amplitude up to $1 \mathrm{~mm}$. The ALS injector system injects beam at a $1 \mathrm{~Hz}$ rate in bursts of $\sim 1 \%$ of the full beam current. The injected beam has $\sim 10 \mathrm{~mm}$ horizontal offset from the design orbit. To damp this, the transverse kick is applied until it is damped below the $1 \mathrm{~mm}$ saturation level of the system whereupon the feedback becomes proportional to beam excursions. In the few milliseconds this requires, $C B$ growth is arrested before it exceeds the $1 \mathrm{~mm}$ limit.

\section{FEedBaCk System Design}

\section{General Description}

A general diagram of the TFB system is shown in Figure 1 . The system is identical in both transverse planes. Two transverse $(x, y)$ pickup signals located $\sim 90^{\circ}$ apart in betatron phase are added together with appropriate coefficients to produce a $90^{\circ}$ betatron phase shift between the pickup signal and the kicker for arbitrary kicker location and betatron tune. The electronics systems consist of two microwave receivers for detecting horizontal and vertical moment $\left(I_{0} \Delta x\right)$, a system (shown as two variable attenuators) for mixing the signals from the two pickup stations, a delay, and a power amplifier for driving the kicker. Detection of the transverse moment rather than position makes the FB damping rate current dependent. Except 


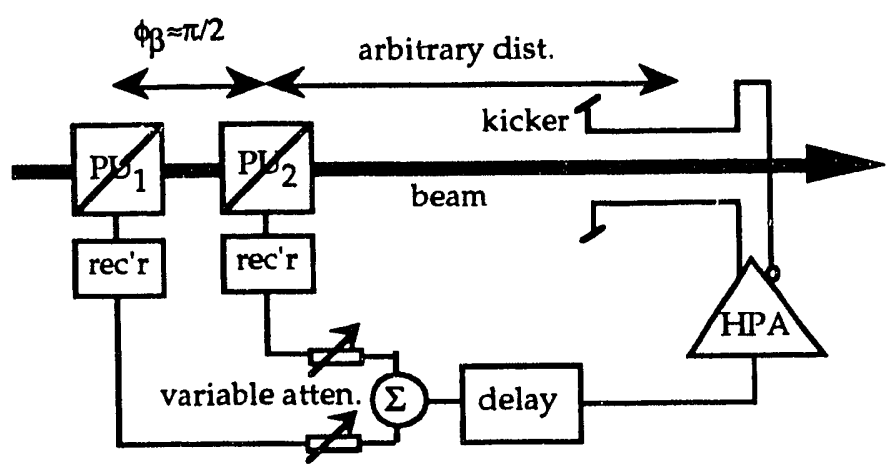

Figure 1: General arrangement of the transverse FB system.

for increased difficulty in calibrating the system, this does not affect the ability of the system to stabilize the beam. Separate amplifiers and kickers are used for each transverse plane. The system uses entirely analog components, although future upgrades include a digital delay. Appropriate interfaces to the accelerator control system are not shown.

\section{Pickups}

A set of four LEP-style beam button pickups[3] are used as the transverse detectors at each pickup location. Each button has a measured pickup impedance of $\sim 1 \Omega$. For their arrangement in the vacuum chamber, transverse pickup impedance is $\sim 1 \Omega / \mathrm{cm}$. With ALS peak bunch currents in excess of $10 \mathrm{~A}$, the pickups provide ample signal. Each button has been loaded with ferrite and reduced in size to suppress high frequency resonances[4] observed in bench measurements. Observations of the button signals during ALS storage ring commissioning show adequate bunchto-bunch isolation to reduce coupling through the TFB system.

In contrast to the kicker operation, position detection is performed at $3 \mathrm{GHz}$ (the sixth harmonic of the RF). This frequency band was chosen because the button pickups are most sensitive in this region. As indicated below, the front end receivers detect beam position at this frequency and subsequently demodulate the position signals to baseband for driving the kickers.

\section{Kickers and Amplifiers}

Each transverse kicker is a pair of $50 \Omega$ quarter-wave striplines at $250 \mathrm{MHz}$ operated in difference mode. The kicker parameters are listed in Table 2.

The power required to damp the amplitude $\Delta x$ is, using $V_{b}$ from Eq. 1, given by

$$
P_{k}=\frac{V_{b}^{2}}{2 R_{k}}
$$

indicating that we require $>250 \mathrm{~W} /$ plane. One drawback of kicking in the baseband is the problem of obtaining power amplifiers (500 W cw each) which have good linearity and phase characteristics over this relatively wide.

\begin{tabular}{clc}
\hline \hline Parameter & Description & Value \\
\hline$Z_{\text {inp }}$ & Input impedance & $50 \Omega$ \\
$2 b$ & Plate separation & $7.2 \mathrm{~cm}$ \\
$\ell$ & Kicker electrical length & $30 \mathrm{~cm}$ \\
$g$ & Coverage factor & 1.1 \\
$R_{k, D C}$ & Kicker impedance at DC & $8.9 \mathrm{k} \Omega$ \\
$R_{k, 250}$ & Kicker impedance at $250 \mathrm{MHz}$ & $3.6 \mathrm{k} \Omega$ \\
$n_{k}$ & Number of kickers/plane & 1 \\
$P_{T}$ & Min. amplifier power & $350 \mathrm{~W}$ \\
\hline \hline
\end{tabular}

Table 2: Transverse stipline kicker parameters.

frequency range (0.1-250 $\mathrm{MHz}$ ). Presently, several manufacturers of such amplifiers have submitted proposals and bids, procurement of one of these is expected to take place during the next several weeks. Available amplifiers are solid-state with $>57 \mathrm{~dB}$ gain.

\section{$R F$ Electronics}

The most intricate, and perhaps most interesting electronics in the system, are the $3 \mathrm{GHz}$ front end receivers, shown in more detail in Figure 2. Bunch to bunch position variations due to coupled bunch instabilities result in amplitude modulation of the sixth harmonic $3 \mathrm{GHz}$ beam signal (carrier) detected by the button pickups. The pickup signals are routed to the receivers via accurately phase-matched coaxial cables. In addition, the receiver inputs employ adjustable delay lines for trimming of the phase match between channels. The button signals are first bandlimited with $\pm 250 \mathrm{MHz}$ bandpass filters centered at $3 \mathrm{GHz}$, allowing for double sideband AM detection. Given the fact that the system bandwidth is limited to $250 \mathrm{MHz}$ (baseband) by the power amplifiers, the bandpass filters were chosen so as not to exceed this bandwidth in order to maximize signal to noise. In addition, the bandpass filters prevent intense spikes from individual bunches from reaching the ensuing gain stages. Apart from the adjustable attenuators, whose function is described below, the remaining portions of the receivers simply sum and difference the button signals to produce $x$ and $y$ position signals. The $x$ and $y$ signals are demodulated to baseband with a $3 \mathrm{GHz}$ local oscillator locked to the storage ring RF. As a practical note, the microwave hybrids for performing sums and differences have been purchased as an integrated package, commonly known as a monopulse comparator. With no significant increase in cost, the integration of the hybrids yields better phase and amplitude balance compared to using separate components. Demodulation components consist of standard mixers and $250 \mathrm{MHz}$ low pass filters.

In order to reject signals due to closed orbit offsets, the receivers employ feedback loops which eliminate slowly varying position signals by setting the gains of each button signal so that the beam appears to be effectively centered in the button array. At the outputs of the $x$ and $y$ channel low pass filters, low frequency error signals are detected via 


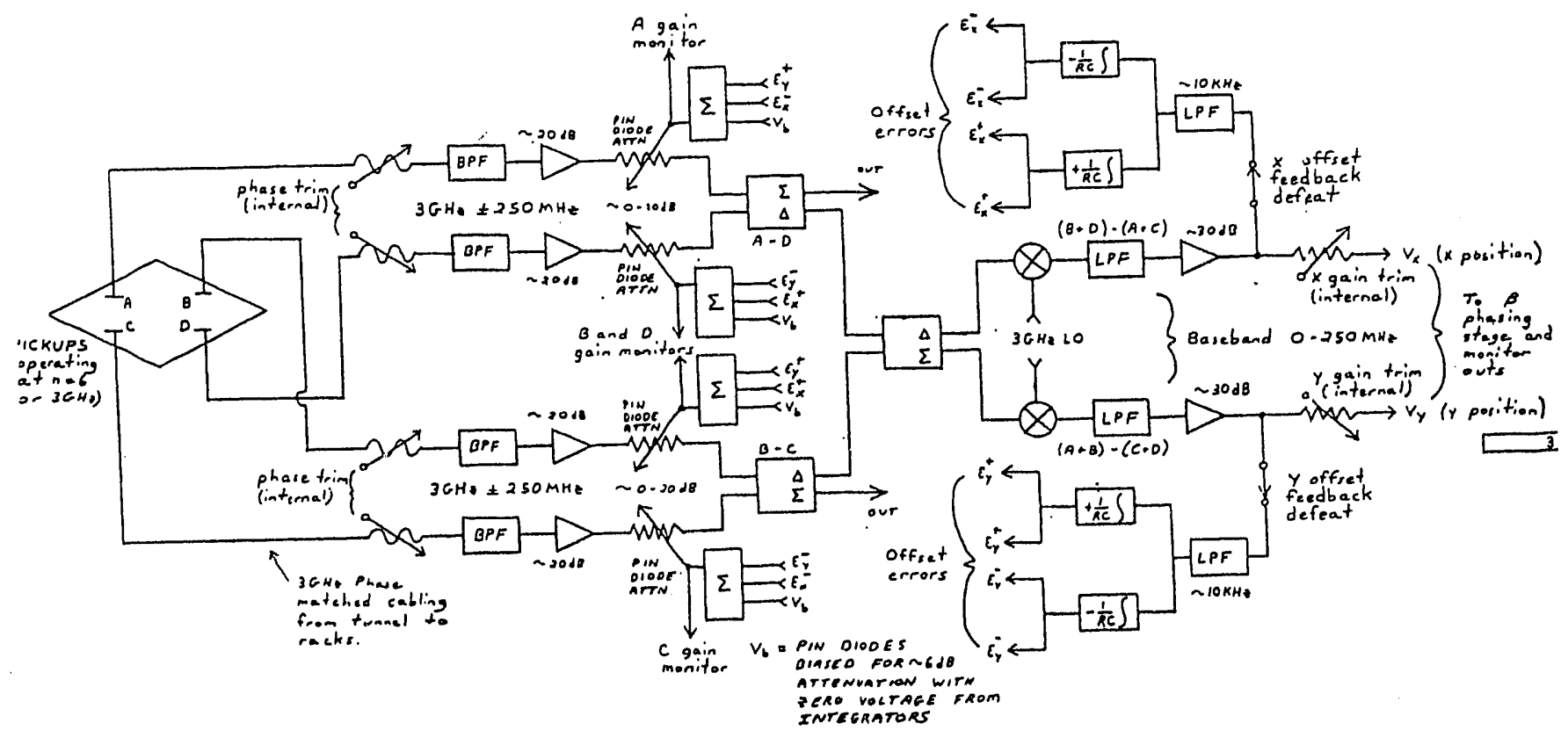

Figure 2: Receivers used in the transverse FB system.

extremely narrow band low pass $(<10 \mathrm{kHz})$ filters. These signals, with the aid of the integrators, are used to drive variable attenuators in the RF legs of the signal paths for each button. The action of the feedback is such that the attenuator settings vary in a way that keeps the $<10 \mathrm{kHz}$ frequency components of the $x$ and $y$ output signals at zero. In addition, the closed-orbit feedback loops are configured as to not change the system gain. The narrow bandwidth of the feedback allows the higher frequency components of the position signals (betatron oscillations) to pass undisturbed to the kickers. With this $10 \mathrm{kHz}$ bandwidth, it is conceivable that position signals due to magnet power supply ripple can be zeroed. The variable attenuators are simply RF mixers configured in "attenuator" mode. That is, the DC coupled IF ports of the mixers are driven by error currents from the integrators esulting in controlled attenuation of the RF signals passing from the RF to LO ports. In this application, it is important that the control current does not greatly alter the phase characteristics of the RF signals. At present, various mixers are being evaluated for this applications.

Returning to Figure 1, the signals from each receiver are combined in proportions dictated by the Twiss parameters at the two pickups and the phase advance between the two pickups and the kicker. These parameters are determined by an on line computer program from quadrapole current measurements. Horizontal and vertical correction signals are then sent to the power amplifiers and kickers via $\sim 1$ tuin delay. For commissioning of the system, the delay (approximately $600 \mathrm{nsec}$ ) will consist of a coaxial cable.

\section{CoNClusions}

We have designed a TFB system to damp coupled-bunch oscillations in the ALS. Currently, RF electronics are being delivered, the amplifiers are being ordered, and the stripline kickers are being constructed for installation in summer of 1993 . The entire system should be installed and operational within a year.

The authors would like to thank the SLAC Feedback Group for many useful discussions and members of the Center for Beam Physics at LBL for their general support and encouragement.

\section{REFERENCES}

[1] 1-2 GeV Synchrotron Radiation Source-CDR, LILL PUB-5172 Rev., July 1986.

[2] J. Byrd and J. Corlett, Study of Coupled-bunch Collective Effects in the $A L S$, these proceedings.

[3] J. Borer, Instrumentation and Diagnostics Used in LEP Commissioning, proceedings of the 1990 Accelerator Instrumentation Workshop, Batavia IL, AIP Proc. 229.

[4] W. Barry, Broad-band Characteristics of Circular Button Pickups, proceedings of the 1992 Accelerator Instrumentation Workshop, Berkeley CA, LBL-32939, UC-406, Oct. 1992. 

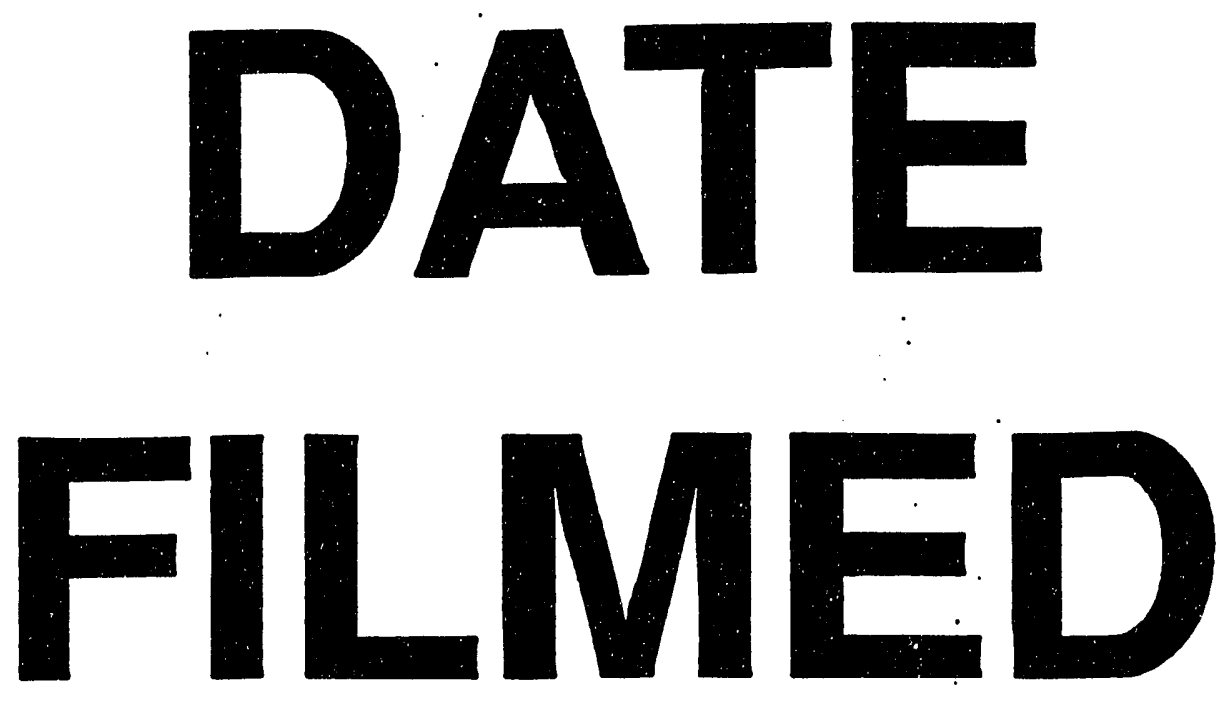

$10 / 20 / 93$
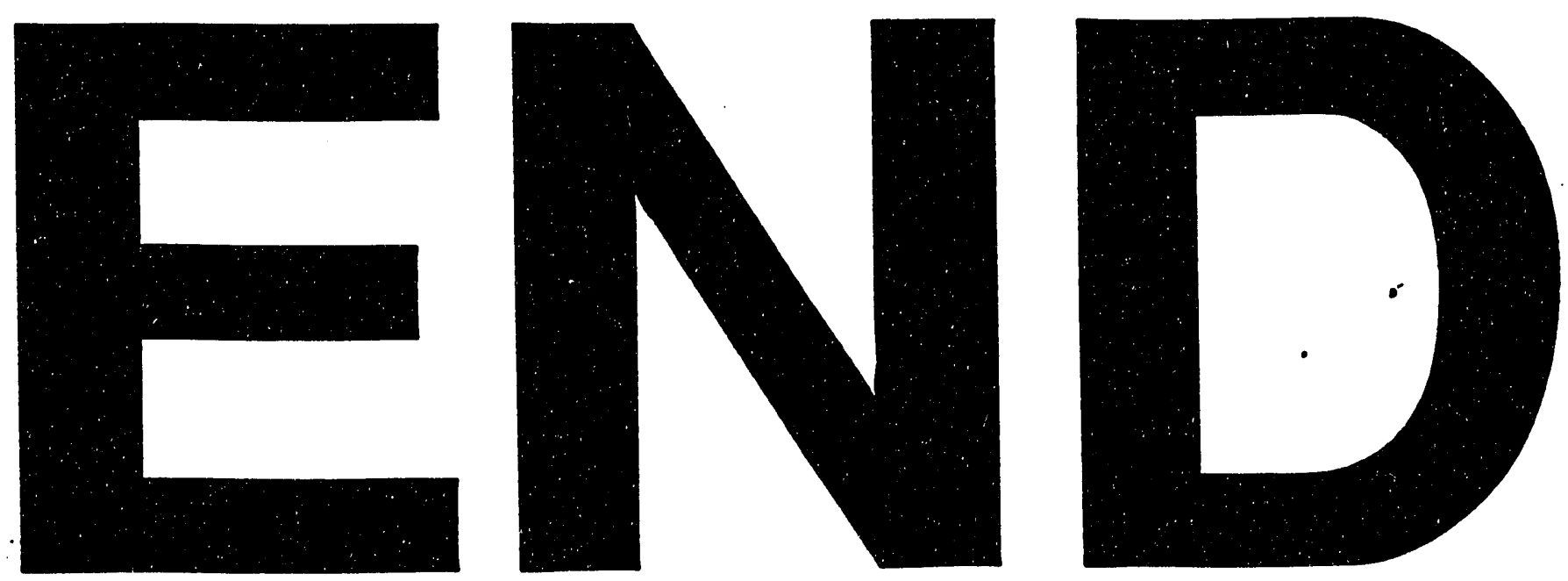
